\title{
ANYONS AND SYSTEMS OF PARTICLES WITH MAGNETIC INTERACTIONS IN THE THERMODYNAMIC LIMIT
}

\author{
W. I. Skrypnik \\ Institute of Mathematics, 3 Tereshchenkivska Str., Kyiv, Ukraine
}

(Received June 14, 1996)

\begin{abstract}
Gibbs states and dynamics are described for systems of charged particles interacting via effective electromagnetic field, characterized by a (singular) vector potential with zero electrostatic component(magnetic interaction). The mean-field type limit for the Gibbs (grand canonical) correlation functions for the 2- $d$ Chern-Simons (CS) system (the vector potential of the $n$-particle system is a skew derivative of the Coulomb potential energy of the $n$-particle system) is performed. It is found that the limit is not unique. For 1- $d$ quantum system of particles with Maxwell-Boltzmann statistics and analog of CS interaction ( the vector potential for the $n$-particle system is the derivative of the 1- $d$ Coulomb potential energy of the system) the Gibbs reduced density matrices are found in the thermodynamic limit. It is shown that in 1- $d$ systems singular interactions may produce anyonic statistics.
\end{abstract}

Key words: Gibbs states, correlation function, thermodynamic limit.

PACS number(s): 05.90.+m

\section{INTRODUCTION}

We consider a system of charged $d$-dimensional particles of $\mathrm{r}$ species interacting via an effective electromagnetic field, which in the case of $\mathrm{n}$ particles is characterized by the vector potential $a_{j}\left(X_{n}\right)=\left\{a_{j}^{\nu}\left(X_{n}\right)\right\}, \nu=1, \ldots, d$, depending on coordinates $X_{n}=\left(x_{1}, \ldots, x_{n}\right) \in \mathbb{R}^{d n}$ of the particles, with zero electrostatic component. It is appropriate to call the interaction magnetic. The classical $\mathrm{n}$ particle system is described by the Hamiltonian $H\left(P_{n}, X_{n}\right)$, given by

$$
H\left(P_{n}, X_{n}\right)=\frac{1}{2} \sum_{j=1}^{n}\left|p_{j}-a_{j}\left(X_{n}\right)\right|^{2},
$$

where $P_{n}=\left(p_{1}, \ldots, p_{n}\right) \in \mathbb{R}^{d n}$ are momenta of particles, $|p|$ denotes the Euclidean norm of the vector $\mathrm{p}$.

$$
a_{j}^{\nu}\left(X_{n}\right)=\sum_{j=1}^{n} a^{\nu}\left(x_{j}-x_{k}\right) e_{j} e_{k}
$$

where $a^{\nu}(x) \in C^{\infty}\left(\mathbb{R}_{0}^{d}\right), \mathbb{R}_{0}^{d}=\mathbb{R}^{d} \backslash 0$ and $\hat{a} C_{0}^{\infty}\left(\mathbb{R}_{0}^{d}\right)=$ $C_{0}^{\infty}\left(\mathbb{R}_{0}^{d}\right), e_{k}$ have the meaning of charges, $e_{k} \in \mathbb{R}$ and $\hat{a}$ is the operator of multiplication by the vectorvalued function $\mathrm{a}(\mathrm{x})$ (pair vector magnetic potential). The last condition on $\mathrm{a}(\mathrm{x})$ means that its singularity is not strong. If the components of this function are integrable(nonintegrable) then the interaction is shortrange(long-range). The Hamiltonian $H\left(P_{n}, X_{n}\right)$ is an unbound function on $\mathbb{R}^{n d} \times \mathbb{R}_{0}^{n d}$ if the function $\mathrm{a}(\mathrm{x})$ is singular, $\mathbb{R}_{0}^{n d}=\mathbb{R}^{n d} \backslash \bigcup_{k<j}\left(x_{j}=x_{k}\right)$.

For quantum $n$ particles with MB(MaxwellBoltzmann) statistics the corresponding Hamiltonian $\dot{H}_{n}$ is an operator defined on a dense set $C_{0}^{\infty}\left(\mathbb{R}_{0}^{n d}\right)$ in
$L^{2}\left(\mathbb{R}^{n d}\right)$

$$
\dot{H}_{n}=\frac{1}{2} \sum_{j=1}^{n}\left(i \frac{\partial}{\partial x_{j}}-a_{j}\left(X_{n}\right)\right)^{2} .
$$

In the case of the smooth functions $a_{j}$ the corresponding quantum Hamiltonian $H_{n}$ has the domain of the $n d-$ dimensional Laplacian. The simplest cases of the singular (smooth) interaction are described by the condition of quasiintegrability (integrability) for $n$-particle system.

$$
\begin{aligned}
& a_{j}\left(X_{n}\right)=\frac{\partial}{\partial x_{j}} U\left(X_{n}\right), \\
& U\left(X_{n}\right)=\sum_{1 \leq k<j \leq n} e_{k} e_{j} \phi\left(x_{j}-x_{k}\right), \\
& X_{n} \in \mathbb{R}_{0}^{n d}\left(X_{n} \in \mathbb{R}^{n d}\right),
\end{aligned}
$$

or $a(x)=\frac{\partial \phi(x)}{\partial x}, x \in \mathbb{R}^{d} \backslash 0,\left(x \in \mathbb{R}^{d}\right)$ i.e. the pair vector magnetic potential $\mathrm{a}(\mathrm{x})$ is a gradient of pair (scalar) magnetic potential $\phi(x)$ (a smooth function), which can be a multivalued function for the singular interaction such that $\exp \{i \phi(x)\}$ is a bona-fide function. It is not difficult to check that

$$
\dot{H}_{n}=\exp \left\{i \hat{U}_{n}\right\} \dot{H}_{n}^{0} \exp \left\{-i \hat{U}_{n}\right\}
$$

where $\hat{U}_{n}$ is the operator of multiplication by the function $U\left(X_{n}\right), \dot{H}_{n}^{0}$ is the minus one-half $n d$-dimensional Laplacian, restricted to $C_{0}^{\infty}\left(\mathbb{R}_{0}^{n d}\right)$. This equality holds for a smooth interaction if the dots over $H_{n}, H_{n}^{0}$ are removed (the interaction is gauged out). There exists the selfadjoint extension $H_{n}$ of $\dot{H}_{n}$, given by the equality

$$
\exp \left\{-t H_{n}\right\}=\exp \left\{i \hat{U}_{n}\right\} \exp \left\{-t H_{n}^{0}\right\} \exp \left\{-i \hat{U}_{n}\right\}
$$




\section{W. I. SKRYPNIK}

where $\exp \left\{-t H_{n}^{0}\right\}$ is the free diffusion semigroup whose infinitesimal generator coincides with the minus one-half nd-dimensional Laplacian. Bose (Fermi) statistics correspond to the case of symmetrical(antisymmetrical) wave functions. There are other selfadjoit extensions which introduce interaction, for example, through $\delta$-pair potential into $H_{n}^{0}$ in the right side of (1.4), but we'll not consider them in what follows.

Our motivation to study the systems with magnetic (singular) interaction, satisfying (1.2), is explained by the fact, that among them there is the remarkable 2$d$ system with CS(Chern-Simons) magnetic interaction, corresponding to case [1]

$$
\begin{aligned}
& U\left(X_{n}\right)=\alpha U_{C S}\left(X_{n}\right)=\alpha \sum_{1 \leq k<j \leq n} \phi_{C S}\left(x_{j}-x_{k}\right), \\
& \phi_{C S}(x)=\arctan \frac{x^{2}}{x^{1}} x=\left(x^{1}, x^{2}\right)=x^{1}+i x^{2} \in \mathbb{C}, \\
& a_{j}^{\nu}\left(X_{n}\right)=\alpha \epsilon^{\nu \gamma} \frac{\partial}{\partial x_{j}^{\gamma}} U_{C}\left(X_{n}\right), \\
& U_{C}\left(X_{n}\right)=\sum_{1 \leq k<j \leq n} e_{j} e_{k} \phi_{C}\left(x_{j}-x_{k}\right), \phi_{C}(x)=\ln |x|,
\end{aligned}
$$

where $\epsilon^{\nu \gamma}$ is the skew symmetrical tensor and the summation is meant over the repeating indices. This system is a result of the reduction, that eliminates the electromagnetic potential from the equation of motion, of the 3- $d$ CS (pure topological) electrodynamics, in which the Lagrangian contains a topological CS term instead of the Maxwellian term. It is evident that for it the condition of quasiintegrability (1.2) holds. It turns out that the operator $\exp \left\{i \hat{U}_{n}\right\}$, which is the operator of multiplication by the function $\exp \left\{i \alpha U_{C S}\left(X_{n}\right)\right\}$, has the remarkable property: it changes the statistics. That is, $\exp \left\{i \alpha \phi_{C S}(x)\right\}=\left(\frac{x}{|x|}\right)^{\alpha}=\epsilon_{0}^{\alpha}(x)$ and the function

$$
\begin{aligned}
& \psi_{\alpha}^{\epsilon}\left(X_{n},(e)_{n}\right)=\exp \left\{i \alpha U_{C S}\left(X_{n}\right)\right\} \psi^{\epsilon}\left(X_{n},(e)_{n}\right) \\
& =\prod_{1 \leq k<j \leq n}\left(\epsilon_{0}\left(x_{j}-x_{k}\right)\right)^{\alpha e_{j} e_{k}} \psi^{\epsilon}\left(X_{n},(e)_{n}\right)
\end{aligned}
$$

satisfies the anyonic statistics $\left(e_{j}\right.$ are charges $)$

$$
\begin{aligned}
& \psi_{\alpha}^{\epsilon}\left(x_{1}, e_{1} ; \ldots ; x_{k}, e_{k} ; \ldots ; x_{j}, e_{j} ; \ldots ; x_{n}, e_{n}\right)= \\
& =\epsilon(-1)^{\alpha e_{j} e_{k}} \psi_{\alpha}^{\epsilon}\left(x_{1}, e_{1} ; \ldots, x_{j}, e_{j} ; \ldots, x_{k}, e_{k} ; \ldots, x_{n}, e_{n}\right)
\end{aligned}
$$

if $\psi_{\epsilon}$ is $\operatorname{symmetrical}(\epsilon=+)$ or antisymmetrical $(\epsilon=-)$. In two dimensions the change of statistics is given by the function $\mathbb{C}$-valued function $\epsilon_{0}(x), x \in \mathbb{C}$. The analog of this function exists in one dimension, namely, $\epsilon(x)$ $=\frac{x}{|x|}=+(-) 1$, if $x>0(x<0)$. So, the anyonic statistics exists in one dimension also, since (1.6) holds if the function $\epsilon(x)$ is put into (1.5) instead of the function $\epsilon_{0}(x)$ and all variables $x_{j}$ are one-dimensional. Equal- ity $\sin \arccos \epsilon(x)=\sqrt{1-\epsilon^{2}}=0$, yields the identity $\epsilon^{\alpha}(x)=\exp \{i \alpha \arccos \epsilon(x)\}=\exp \left\{i \alpha \varphi_{*}(x)\right\}$. It is clear that the operator of differentiation commutes with the operator of multiplication by the function $\epsilon(x)$ on $C_{0}^{\infty}(\mathbb{R} \backslash 0)$. From this it follows that $n$-dinensional Laplacian commute with the operator $\exp \left\{i \alpha \hat{U}_{* n}\right\}$ on $C_{0}^{\infty}\left(\mathbb{R}_{0}^{n}\right)$, where $\hat{U}_{* n}$ is the operator of multiplication by $U_{*}\left(X_{n}\right)=\sum_{1 \leq k<j \leq n} \varphi_{*}\left(x_{j}-x_{k}\right)$. As a result, the operator $H_{* n}^{(\alpha)}=\exp \left\{i \alpha \hat{U}_{* n}\right\} H_{n}^{0} \exp \left\{-i \alpha \hat{U}_{* n}\right\}$ is the selfadjoint extension of $\dot{H}_{n}^{0}$ from $C_{0}^{\infty}\left(\mathbb{R}_{0}^{n}\right)$ with an obvious domain. This Hamiltonian, restricted to the subspaces of symmetrical or antisymmetrical functions, describes anyons in one dimension. The derivative of $\varphi_{*}(x)$ does not exist and there are no corresponding collective electromagnetic potentials $a_{j}\left(X_{n}\right)$.

1- $d$ anyons for $\alpha=1$ and one sort of particles with the unit charge have predecessors, namely, impenetrable bosons, introduced by Girardau in 1961 [2]. The absence of a generalized condensation for 1- $d$ impenetrable bosons was proved by Schultz [3] (the grand partition function for the impenetrable bosons coincides with the grand partition function of free fermions).

The case of 2- $d$ anyons is very interesting for physicists who expect that anyonic condensation (anyons can create clusters that are either bosons or fermions), if it exists, looks like Bose condensation and can be used as an explanation of a high temperature superconductivity [4-5]. Up till now only lattice approximation for anyons were treated rigorously in the framework of Lattice Electrodynamics(Maxwellian term is not neglected, so, roughly speaking, the anyons interact via electrostatic forces) [6]).

Quantum system with CS magnetic interaction and MB statistics loses properties, connected with phase transitions, since the grand partition function for it coincides with the grand partition function of the free particle systems. But if one is interested in the behavior of reduced density matrices for quantum systems with the Bose or Fermi statistics in some scaling limit, for which the role of the statistics in qoestion is negligible, then it is desirable to find the limit of reduced density matrices for the quantum systems with MB statistics and, even, that of correlation functions for the classical systems.

There is a remarkable example of $1-d$ system with CS-type magnetic interaction, corresponding to the 1$d$ Coulomb magnetic interaction, for which $\phi(x)=\lambda|x|$. The 1- $d$ anyons, interacting through this pair magnetic potential can be considered as an analog of the $2-d$ free anyons. It is interesting that such a system can be derived in the same way from the $2-d$ quasi-topological Electrodynamics as the CS particle system from the 3-d Electrodynamics (the term $A_{0} \partial_{1} A_{1}$ is considered in the Lagrangian instead of the Maxwellian term).

So, in order to describe anyonic systems in two dimensions and their analogs in one dimension from different angles we have to consider at first the problems, arising from considering the classical and quantum systems with different magnetic interactions and usual statistics in the thermodynamic limit. 
It turns out that the problem of the thermodynamic limit for classical dynamics, for Gibbs(grand canonical) correlation functions and Gibbs reduced density matrices for systems with magnetic long-range interactions is nontrivial even for the simplest cases((1.2), (1.4) hold). We demonstrate that the Gibbs correlation functions in the mean-field type limit for classical system with the CS magnetic interaction and the reduced density matrices for 1- $d$ system with Coulomb pair magnetic potential and MB statistics have unexpected properties: in the first case the limit is not unique and in the second on the diagonal the functions coincide with the reduced density matrices of free particle systems and off the diagonal they are non-zero if the differences of special variables sit on the lattice. We came to the conclusion that long-range interaction may be unremovable by the gauge transformation in the thermodynamic limit.

\section{CLASSICAL DYNAMICS}

The equation of motion for integrable classical $n$ particle system $\left(a_{j}\right.$ satisfies the condition (1.2) with a smooth function $\mathrm{U}$ ) is given by [7]

$$
\begin{aligned}
& \dot{x}_{j}(t)=\frac{\partial_{j} H\left(P_{n}, Q_{n}\right)}{\partial x_{j}}=m^{-1}\left(p_{j}-a_{j}\left(X_{n}\right)\right) \\
& \dot{p}_{j}(t)=-\frac{\partial H\left(P_{n}, Q_{n}\right)}{\partial p_{j}} \\
& =m^{-1} \sum_{k=1}^{n}\left(\left[p_{k}-a_{k}\left(X_{n}\right)\right], \partial_{j} a_{k}\left(X_{n}\right)\right) .
\end{aligned}
$$

where the dot over variables in the lefthandside of the equation means time derivative, the partial derivative in $x_{j}$ we often denote by $\partial_{j}$ and $(.,$.$) is the scalar product$ of the $d$-dimensional Euclidean space.

Now, let the condition of integrability hold

$$
\partial_{j} a_{k}\left(X_{n}\right)=\partial_{k} a_{j}\left(X_{n}\right), a_{j} \in C^{\infty}\left(\mathbb{R}^{n d}\right)
$$

It is not difficult to check that the generalized velocities $v_{j}=p_{j}-a_{j}\left(X_{n}\right)$ commute with respect to the Poisson bracket

$$
\begin{aligned}
& \left\{v_{j}, v_{k}\right\}=\sum_{l=1}^{n}\left[\left(\frac{\partial_{l} v_{j}}{\partial x_{l}}, \frac{\partial v_{k}}{\partial p_{l}}\right)-\left(\frac{\partial v_{j}}{\partial p_{l}}, \frac{\partial v_{k}}{\partial x_{l}}\right)\right] \\
& =\partial_{j} a_{k}\left(X_{n}\right)-\partial_{k} a_{j}\left(X_{n}\right)=0
\end{aligned}
$$

From $\left\{v_{j}^{2}, v_{k}\right\}=2 v_{j}\left\{v_{j}, v_{k}\right\}=0$ it follows that $\left\{H, v_{j}\right\}=$ 0 , i.e. the functions $v_{j}$ are integrals of motion. From the first equation of motion it follows that

$$
x_{j}(t)=x_{j}+m^{-1} t\left(p_{j}-a_{j}\left(X_{n}\right)\right)
$$

where $\left(p_{j}, x_{j}\right)$ are initial momenta and coordinates. Substituting the equation for $x_{j}$ and (2.1) into the equation for momenta we obtain

$$
\dot{p}_{j}(t)=\sum_{k=1}^{n} \dot{x_{k}}(t) \partial_{k} a_{j}\left(X_{n}\right)=\dot{a}_{j}\left(X_{n}\right)
$$

Hence the solution of the $n$-particle equation of motion is given by

$$
\begin{aligned}
& x_{j}(t)=x_{j}+m^{-1} t\left(p_{j}-a_{j}\left(X_{n}\right)\right), \\
& p_{j}(t)=p_{j}+a_{j}\left(X_{n}(t)\right)-a_{j}\left(X_{n}\right) .
\end{aligned}
$$

The equation of motion for the infinite particle system is written as follows

$$
\begin{aligned}
& \dot{x}_{j}(t)=m^{-1}\left(p_{j}(t)-a_{j}(X(t)), j>0,\right. \\
& \dot{p}_{j}(t)=m^{-1} \sum_{k>0}\left(\left[p_{k}(t)-a_{k}(X(t)] \partial_{j} a_{k}(X(t)),\right.\right.
\end{aligned}
$$

where $P=\left(p_{1}, \ldots, p_{n}, ..\right), X=\left(x_{1}, \ldots, x_{n}, \ldots.\right)$, $a_{j}(X)=\sum_{l>0} e_{j} e_{l}(\partial \phi)\left(x_{j}-x_{l}\right)$. Let $\hat{\mathbb{R}}^{d}$ be the space $\mathbb{R}^{\infty d} \times$ $\mathbb{R}_{f}^{\infty d}$, where $\mathbb{R}_{f}^{\infty}$ is the space of locally finite configurations from the infinite Cartesian product of $\mathbb{R}^{d}$. It is not difficult to see that the Hamiltonian diverges on the above space, but the equation of motion makes sense if $a_{j}(X)$ are finite for all $\mathrm{j}$. Let us assume that $\phi$ is strictly short-range. Then the infinite sum, defining $a_{j}(X)$ is convergent for the initial sequence $\mathrm{X}$ from $\mathbb{R}_{f}^{\infty d}$. If for every moment the right side of the equation of motion for momenta is well defined (a collapse is excluded) then the equality holds $\dot{p}_{j}(t)=\dot{a}_{j}(X(t))$, so the sequence of generalized velocities are integrals of motion. From the first equation of motion it follows now that $x_{j}(t)=$ $x_{j}+m^{-1} t\left(p_{j}-a_{j}(X)\right)$. And we see that the following formulae define the solution of the equation of motion for the infinite particle system

$$
x_{j}(t)=x_{j}+m^{-1} t\left(p_{j}-a_{j}(X)\right)
$$

$$
p_{j}(t)=p_{j}+a_{j}(X(t))-a_{j}(X)
$$

It is clear that the transformation $\mathrm{Z}$, defined by $Z(P, X)=\left(P-A_{\phi}, X\right)$, reduces the solution to the free particle dynamics on $\mathbb{R}^{\infty d} \times \mathbb{R}_{f}^{\infty d}$, where $A_{\phi}$ is the sequence $\left\{a_{j}\right\}$. It is evident, also, that for long-range forces it is difficult to figure out what are the configurations for which $a_{j}(X)$ and $a_{j}(X(t))$ are finite. It is one of the reasons to consider the BBGKY hierarchy [8]. 


\section{W. I. SKRYPNIK}

\section{CLASSICAL GIBBS SYSTEMS AND MEAN-FIELD TYPE LIMIT}

Let us consider Gibbs (grand-canonical) correlation functions for the $d$-dimensional system in a compact domain $\Lambda$, defined by

$$
\rho^{\Lambda}\left(P_{m}, X_{m} ;(e)_{m}\right)=z_{(e)_{m}} \Xi_{\Lambda}^{-1} \sum_{n \geq 0}(n !)^{-1} \sum_{\left(e^{\prime}\right)_{n}} \exp \left\{\beta \mu\left(\left(e^{\prime}\right)_{n}\right)\right\} \int_{\Lambda} d P_{n}^{\prime} d X_{n}^{\prime} \exp \left\{-\beta H\left(P_{m}, P_{n} ; X_{m}, X_{m}^{\prime}\right)\right\}
$$

where

$$
\mu\left((e)_{n}\right)=\sum_{k=1}^{n} \mu_{e_{k}}, \quad \Xi_{\Lambda}=\exp \left\{V(\Lambda) \sum_{e} z_{e}\right\}, z_{(e)_{m}}=\prod_{s=1}^{m} z_{e_{s}},
$$

$\mu_{e}, z_{e}$ are chemical potential and activity of the particles with the charge e, correspondingly, $V(\Lambda)$ is the volume of the domain $\Lambda$. integrating by $P_{n}^{\prime}$ is performed over $\mathbb{R}^{n d}$ and summation by $e^{\prime}$ is over $E_{c}\{r\}$ that is a set of r elements on a line. We omit the dependence of $\mathrm{H}$ on $(e)_{n}$ in our notations.

Now, integrating over $P_{n}^{\prime}$, we obtain

$$
\rho^{\Lambda}\left(P_{m}, X_{m} ;(e)_{m}\right)=z_{(e)_{m}} \Xi_{\Lambda}^{-1} \sum_{n \geq 0}(n !)^{-1} \sum_{\left(e^{\prime}\right)_{n}} z_{\left(e^{\prime}\right)_{l}} \int_{\Lambda} d X_{n}^{\prime} \exp \left\{-\frac{\beta}{2} \sum_{k=1}^{m}\left|p_{k}-a_{k}\left(X_{m}, X_{n}^{\prime}\right)\right|^{2}\right\}
$$

Using the Fourier transform in momentum variables, we obtain

$$
\rho_{\Lambda}\left(P_{m}, X_{m} ;(e)_{m}\right)=z_{(e)_{m}}(2 \pi)^{-\frac{m d}{2}} \int d Q_{m} \exp \left\{-\frac{\left|Q_{m}\right|^{2}}{2}\right\} \exp \left\{i \sqrt{\beta} \sum_{k=1}^{m}\left[\left(q_{k}, p_{k}\right)+e_{k} a_{m}^{*}\left(x_{k}\right)\right]\right\} \hat{\rho}^{\Lambda}\left(Q_{m}, X_{m}\right)
$$

where

$$
\begin{aligned}
& \hat{\rho}^{\Lambda}\left(Q_{m}, X_{m}\right)=\exp \left\{\sum_{e} z_{e} \int_{\Lambda}\left(\exp \left[i \sqrt{\beta} e a_{m}^{*}(x)\right]-1\right) d x\right\} \\
& a_{m}^{*}(x)=\sum_{k=1}^{m}\left(q_{k}, a\left(x-x_{k}\right)\right) e_{k}
\end{aligned}
$$

$(.,$.$) is the scalar product of the d$-dimensional Euclidean space, and if $x=x_{j}$ then the term, corresponding to $k=j$, is not taken into account in the expression for $a_{m}^{*}(x)$.

It is evident that we can pass to the thermodynamic limit $\Lambda \rightarrow \mathbb{R}^{d}$ if the function $|a(x)|$ is integrable. The functions

$$
\rho\left(P_{m}, X_{m} ;(e)_{m}\right)=\lim _{\Lambda} \rho^{\Lambda}\left(X_{m}, P_{m} ;(e)_{m}\right)
$$

in this case are expressed through formula (3.1) in which instead of the functions $\hat{\rho}^{\Lambda}$ their limits are present(trivial estimate shows that they exist).

The problem of the existence of the Gibbs correlation functions for classical systems with long-range magnetic interactions in the thermodynamic limit remains opened. It can be solved for some cases in the mean-field type limit.

By mean-field type limit we mean the limit $\varepsilon \rightarrow 0$ for the correlation functions $\rho_{\varepsilon}$ with rescaled activity $\left(\frac{z}{\epsilon}\right)$, compact domain $B_{R(\varepsilon)}$, where $B_{R}$ is a sphere of radius $\mathrm{R}$, vector magnetic potential $a^{\varepsilon}(x)=\varepsilon^{\gamma} a_{\varepsilon}(x)$ (for the case $a(x)=\frac{a^{0}(x)}{|x|^{-2 s}}, s>0, a^{0}$ is a regular function, $a_{\varepsilon}(x)=\frac{a^{0}(x)}{\left(|x|^{2}+\lambda^{2}(\varepsilon)\right)^{s}}, \lambda(\varepsilon)=\varepsilon^{\nu}$.

For the usual systems of particles when, instead of the magnetic potential $\mathrm{a}(\mathrm{x})$, a usual pair potential is rescaled, by multiplying by $\varepsilon$, or in the same way the inverse temperature $\beta$, the mean field limit corresponds to a high temperature low density limit. For the magnetic interaction the same is true: if the inverse temper- 
ature is rescaled by $\varepsilon^{2 \gamma}$ and $a_{\varepsilon}(x)$ is used instead of $a^{\varepsilon}(x)$ then the same result is obtained, if the former rescaling is used and the momenta are rescaled also $\left(\varepsilon^{-\gamma} P_{m}\right)$ (in [9] the mixed case of rescaling was considered and the corresponding rescaling of momenta is meant in the definition of the rescaled correlation functions $\rho_{\varepsilon}$ ). For the usual systems with a regular short-range potential, if a rescaled temperature tends to zero faster than $\varepsilon$, than the interaction dies out in the mean-field limit. The same is also true for a regular short-range pair magnetic interaction, as it is seen from the above representation. If the square of the singular short-range potential diverges than a stronger rescaling of the temperature is necessary to kill the divergence.

In the following theorem we show that for the longrange CS magnetic interaction the mean-field type limit exists for $\rho_{\varepsilon}$ only if the power of $\varepsilon$ in the rescaled temperature is greater than unity. We also show that the limit is not unique and depends on the way $R(\varepsilon)$ tends to infinity.

Let us consider the CS case, i.e.d $=2$ and $a^{0 \nu}(x)=$ $\sum_{\sigma=1}^{2} x^{\sigma} \epsilon^{\sigma \nu}, \gamma=\frac{3}{2}$ and the temperature is not rescaled(this rescaling is equivalent to the rescaling of [9])

\section{Theorem 1}

Let $\nu<\frac{1}{2}$ and $\sum_{e} z_{e} e=0$ (neutrality). If

1) $\lim _{\varepsilon \rightarrow 0} \varepsilon^{2} \ln R(\varepsilon)=0$, then

$$
\lim _{\varepsilon \rightarrow 0} \varepsilon^{m} \rho_{\varepsilon}\left(P_{m}, X_{m} ;(e)_{m}\right)=z_{(e)_{m}} \exp \left\{-\frac{\beta}{2}\left|P_{m}\right|^{2}\right\}
$$

2)if $R(\varepsilon)=\exp \left\{r_{0} \varepsilon^{-2}\right\}$, then

$\lim _{\varepsilon \rightarrow 0} \varepsilon^{m} \rho_{\varepsilon}\left(P_{m}, X_{m} ;(e)_{m}\right)$

$=z_{(e)_{m}}(2 \pi)^{-m} \int d Q_{m} \exp \left\{-\frac{\left|Q_{m}\right|^{2}}{2}+i \sqrt{\beta} \sum_{k=1}^{m}\left(q_{k}, p_{k}\right)\right\}$ $\times \exp \left\{-\left(\sum_{e} z_{e}^{2} e\right) \beta r_{0} B\left(Q_{m}(e), Q_{m}(e)\right)\right\}$

$Q_{m}(e)=2 \sum_{j=1}^{m} q_{j} e_{j}$

where $B(\psi, \psi)=\int_{0}^{2 \pi}\left(\psi_{2} \cos (\varphi)+\psi_{1} \sin (\varphi)\right)^{2} d \varphi$.

A similar result can be obtained for regular long-range potentials $\phi$, whose fourth power is an integrable function, demanding that the expression

$$
\varepsilon^{\gamma} \int_{B_{R(\varepsilon)}} \phi^{2}(x) d x
$$

has a limit as $\varepsilon$ approaches zero. A special care is needed if the potential is singular: it is necessary to estimate short-distance and long-distance divergence of integrals of powers of the potential.

\section{QUANTUM SYSTEMS}

Now we consider the system in the compact domain $\Lambda$ with the Dirichlet boundary condition on its boundary, i.e.with the Hamiltonian $H_{n, \Lambda}$

$$
\begin{aligned}
& P_{n, \Lambda}^{t}=\exp \left\{-\beta H_{n, \Lambda}\right\} \\
& =\exp \left\{i \hat{U}_{n}^{*}\right\} P_{0(n, \Lambda)}^{t} \exp \left\{-i \hat{U}_{n}^{*}\right\}
\end{aligned}
$$

where the semigroup $P_{0(n, \Lambda)}^{t}$ is generated by the $n$ dimensional Laplacian with the Dirichlet boundary condition on the boundary of $\Lambda$. Let us define the reduced density matrices for our systems of $\mathrm{r}$ sorts of particles with the M-B statistics.

$$
\rho^{\Lambda}\left(X_{m} \mid Y_{m}\right)=\Xi_{\Lambda}^{-1} z_{(e)_{m}} \sum_{n \geq 0}(n !)^{-1} \sum_{e_{1}^{\prime}, \ldots, e_{n}^{\prime}} z_{\left(e^{\prime}\right)_{m}} \int_{\Lambda} P_{(\Lambda)}^{\beta}\left(X_{m}, X_{n}^{\prime} \mid Y_{m}, X_{n}^{\prime}\right) d X_{n}^{\prime}
$$

where $\Xi_{\Lambda}$ coincides with the numerator in (1.4) for the case $\mathrm{m}=\mathrm{o}$, the sums in $e_{j}^{\prime}$ are performed over the set $E_{c}\{r\}$, $z_{e}$ is the activity of the particle with the "charge" $e, \beta$ is the inverse temperature, $P_{(\Lambda)}^{\beta}\left(X_{n} \mid Y_{n}\right)$ is the kernel of the operator $P_{n, \Lambda}^{\beta}$. The reduced density matrices in our case are functions in $e_{1}, \ldots, e_{m}$, since the Hamiltonian is diagonal in variables that describe the inner degree of freedom. In order to simplify notations we do not indicate this dependence in $\rho^{\Lambda}$. The following equality is true

$$
\begin{aligned}
\rho^{\Lambda}\left(X_{m} \mid Y_{m}\right) & =\exp \left\{i\left[U^{*}\left(X_{m}\right)-U^{*}\left(Y_{m}\right)\right]\right\} z_{(e)_{m}} P_{0(\Lambda)}^{\beta}\left(X_{m} \mid Y_{m}\right) \exp \left\{G_{\Lambda}\left(X_{m}, Y_{m}\right)\right\}, \\
G_{\Lambda}\left(X_{m}, Y_{m}\right) & =\sum_{e} z_{e} \int_{\Lambda}\left\{\exp \left\{i\left[\sum_{j=1}^{m} e e_{j}\left(\phi\left(x_{j}-x\right)-\phi\left(y_{j}-x\right)\right]\right\}-1\right\} P_{0(\Lambda)}^{\beta}(x \mid x) d x .\right.
\end{aligned}
$$




\section{W. I. SKRYPNIK}

where $P_{0(\Lambda)}^{\beta}(x \mid y)$ is the integral over the Wiener measure concentrated on paths, starting from $\mathrm{x}$ and arriving in $\mathrm{y}$ at the moment $\beta$, of the characteristic function of paths that are inside $\Lambda, P_{0(\Lambda)}^{\beta}\left(X_{m} \mid Y_{m}\right)=\prod_{s=1}^{m} P_{0(\Lambda)}^{\beta}\left(x_{s} \mid y_{s}\right)$.

If $\phi$ is an integrable function than it is easy to pass to the thermodynamic limit in the above expression. The limiting density matrices for a regular short-range magnetic potential $\phi$ are given by

$$
\begin{aligned}
& \rho\left(X_{m} \mid Y_{m}\right)=\exp \left\{i\left[U\left(X_{m}\right)-U\left(Y_{m}\right)\right]\right\} z_{(e)_{m}} P_{0}^{\beta}\left(X_{m} \mid Y_{m}\right) \exp \left\{G\left(X_{m}, Y_{m}\right)\right\} \\
& G\left(X_{m}, Y_{m}\right)=(2 \pi \beta)^{-\frac{d}{2}} \sum_{e} z_{e} \int\left\{\exp \left\{i\left[\sum_{j=1}^{m} e e_{j}\left(\phi\left(x_{j}-x\right)-\phi\left(y_{j}-x\right)\right]\right\}-1\right\} d x .\right.
\end{aligned}
$$

where $P_{0}^{\beta}(x \mid y)$ is the kernel of the semigroup generated by the one-half $d$-dimensional Laplacian and the integration is performed over the $d$-dimensional space. In general it is unclear whether it is possible to use the formulae for long-range potentials in the thermodynamic limit.

Let us consider the $1-d$ system with the potential $\phi(x)=\lambda|x|$ on the interval $\Lambda=[-\mathrm{L}, \mathrm{L}]$. It turns out that only for such a system from the systems with long-range magnetic interactions we can easily pass to the thermodynamic limit in the expression for $\rho^{\Lambda}=\rho^{[L]}, G_{L}=G_{\Lambda}$. Let us put $l_{+(-)}=\max (\min )\left(X_{m}, Y_{m}\right)$. Then

$$
\begin{aligned}
& G_{L}\left(X_{m}, Y_{m}\right)=\sum_{e} z_{e}\left\{\int_{l_{-}}^{l_{+}}\left(\exp \left\{i e \sum_{s=1}^{m} e_{s} \lambda\left[\left|x-x_{j}\right|-\left|x-y_{j}\right|\right]\right\}-1\right) P_{0(L)}^{\beta}(x \mid x) d x\right. \\
& +\left(\exp \left\{i e \sum_{s=1}^{m} e_{s} \lambda\left[x_{s}-y_{s}\right]\right\}-1\right) \int_{-L}^{l_{-}} P_{0(L)}^{\beta}(x \mid x) d x+\left(\exp \left\{-i e \sum_{s=1}^{m} e_{s} \lambda\left[x_{s}-y_{s}\right]-1\right) \int_{l_{+}}^{L} P_{0(L)}^{\beta}(x \mid x) d x\right\}
\end{aligned}
$$

It is clear that last two integrals are zero if $e_{j} \in \gamma \mathbb{Z}$ and

$$
x_{j}-y_{j} \in 2 \pi \lambda^{-1} \gamma^{-2} \mathbb{Z}
$$

If the former condition is not satisfied then the integrals tens to $-\infty$ and the density matrices tend to zero in the thermodynamic limit $(\mathrm{L}$ tends to $\infty)$. As a result the following theorem is true

\section{Theorem 2}

Let $e_{j} \in \mathbb{Z}$. Then, if the condition (4.4) is satisfied, the reduced density matrices for $1-d$ system with the pair magnetic potential $\phi(x)=\lambda|x|$ in the thermodynamic limit are given by (4.3) in which

$$
\begin{aligned}
& G\left(X_{m}, Y_{m}\right)=(2 \pi \beta)^{-\frac{1}{2}} \\
& \times \sum_{e} z_{e} \int_{l_{-}}^{l_{+}}\left\{\exp \left\{i e \sum_{s=1}^{m} e_{s} \lambda\left[\left|x-x_{j}\right|-\left|x-y_{j}\right|\right]\right\}-1\right\} d x
\end{aligned}
$$

where $l_{+(-)}=\max (\min )\left(X_{m}, Y_{m}\right)$. If the condition (4.4) is not satisfied, then the reduced density martices $\rho^{[L]}$ tend to zero for $L \rightarrow \infty$.

We can show that the analog of this theorem holds for systems with the Bose and Fermi statistics for small values of activities(to be published). We expect that our results can also be generalized to the case of impenetrable bosons in one dimension which are simplest examples of 1- $d$ anyons.

\section{ACKNOWLEDGMENTS}

The author is grateful to the Organizing Committee of the $28^{t h}$ Torun Symposium on Mathematical Physics for inviting him to the Symposium where the above results were reported in the fall of 1995 . The author is grateful also to profs. J. T. Lewis and L. A. Pastur for discussions. 
[1] R. Jackiw, S-Y Pi, Phys. Rev. D 15, 3500 (1990).

[2] M. Girardeau, J. Math. Phys. 1, 516 (1960).

[3] T. Schults, J. Math. Phys. 4, 666 (1963).

[4] E. Fradkin, Field theories of condensed matter systems (Addison-Wesley Publishing Company, 1991).

[5] J. Lykken, J. Sonnenschein, N. Wess, Preprint TAUP1858-91.

[6] J. Frohlich., P. Marchetti, Commun. Math. Phys. 121,
177 (1989).

[7] W. Skrypnik, Preprint DIAS STP-92-12.

[8] W. Skrypnik, Ukrainian Math. Journ. 47, 853 (1995).

[9] W. Skrypnik, Ukrainian Phys. Journ. 40, 133 (1995).

[10] W. Skrypnik, Ukrainian Math. Journ. 47, 1686 (1995).

[11] W. Skrypnik, Preprint, DIAS-95-21 (to be published in "Math.Physics, Analysis, Geometry").

\title{
АНІОНИ І СИСТЕМИ ЧАСТИНОК З МАГНЕТНИМИ ВЗАЄМОДІЯМИ У ТЕРМОДИНАМІЧНІЙ ГРАНИЩ
}

\author{
B. I. Скрипник \\ Інститут математики, Украӥна, 252000, Київ, вул. Терешенківсъка, 3
}

\begin{abstract}
Описано Гіббсові стани та динаміку для систем заряджених частинок, що взаємодіють через ефективне електромагнетне поле, що характеризується (сингулярним) векторним потенціялом з нульовою електростатичною компонентою (магнетна взаємодія). Виконане наближення типу середнього поля для гіббсових кореляційних функцій (у великому канонічному ансамблі) для 2- $d$ системи Черна-Сімона (векторний потенціал $n$-частинкової системи є похідною кулонівської потенціяльної енергії $n$-частинкової системи).

Знайдено, що ця границя не є єдина. Для 1- $d$ квантової системи частинок зі статистикою МаксвелаБольцмана і аналогом взаємодії Черна-Сімона (векторний потенціял системи $n$-частинок є похідною 1 -d кулонівської потенціяльної енергії системи) в термодинамічній границі отримано приведені матриці густини Гіббса. Показано, що в 1-d системах сингулярні взаємодії можуть спричиняти аніонну статистику.
\end{abstract}

